Am einfachsten gestaltet sich die Rechnung, wenn man für die vier in die Formeln eingehenden Glieder Tafeln entwickelt, was mit nur geringer Mühe verbunden ist. Man kann dann auch mit einem Blicke übersehen, wie weit diese abgekürzten Gleichungen Gültigkeit haben, und wann die vollständigen Formeln in Anwendung zu bringen sind.

Ich habe mit dem hier beschriebenen Mikrometer eine Reihe von Positionsbestimmungen an Nebelflecken ausgeführt, welche ein günstiges Zeugniss für die Genauigkeit ablegen, die sich mit diesem Apparate auch bei schwierigeren
Objecten erreichen lässt. Die ausführlichen Resultate dieser Messungen sollen in einer der nächsten Publicationen des Astrophysikalischen Observatoriums mitgetheilt werden. Nicht minder günstig äussert sich Boguslawski in der Mittheilung über sein Mikrometer (a. a. O.), wo er angiebt, dass dasselbe in Verbindung mit einem zweifüssigen Cometensucher, bei einer Vergrösserung von 24 bis 28 , mit seinem $3 \frac{1}{2}$ füssigen Heliometer concurriren konnte bei Anwendung einer 40 bis I 4 o fachen Vergrösserung.

Potsdam, Astrophysikal. Observatorium, 1888 März 2.

\title{
Die Bahn des Planeten (183) Istria.
}

In Veranlassung des Aufsatzes des Hrn. Dr. W. Luther in den Astr. Nachr. Nr. $283_{1}$ erlaube ich mir, hier einige Bemerkungen über diesen Planeten mitzutheilen, welche zum Theil einer kleinen Schrift entnommen sind, die soeben als Abdruck aus der, Öfversigt af Finska Vetensk.-Societens Förhandlingare $\mathrm{Bd}$. XXX erschienen ist und in welchen ich eine kurze Zusammenstellung von dem gegeben habe, was bisher in Bezug auf diesen Planeten gerechnet worden ist.

Die relativ grosse Annäherung des Planeten an die Erde bei der Opposition Ende I886 und die dadurch gesteigerte Hoffnung auf eine Wiederentdeckung desselben veranlasste mich im Herbst 1886 eine definitive Bahnbestimmung vorzunehmen. Die daraus hervorgehenden wahrscheinlichsten Elemente, bei welchen $\mu=757.83^{67}$ sich ergab, nebst einer darauf gegründeten Ephemeride von 1886 Oct. 23 bis Nov. 24 und zweier anderen für $\mu=755^{\prime \prime} 79$ und 759".88, welche die zunächst etwa einzuhaltenden Grenzen der Aufsuchungszone angaben, wurden den Herren Dr. V. Knorre und Dr. J. Palisa zugestellt, welche sich im Voraus bereit erklärt hatten, einige Mühe auf das Aufsuchen ver. wenden zu wollen. - Wegen der zu südlichen Declinationen war in Berlin wenig Erfolg zu hoffen und ist das Suchen dort auch ganz unterlassen worden. In Wien hat Dr. Palisa dagegen die ganze Zone, leider ohne erwünschtes Resultat, durchsucht; derselbe sprach die Absicht aus, im Dec. 1886, wenn der Planet in nördlichere Decl. gekommen sei, weitere Nachforschungen anzustellen. Inzwischen waren mir, zum grossen Theile durch Herrn Dr. Knorre, einige weitere in den Nachweisungen für die Planeten im Berliner Jahrbuch nicht citirte Neubestimmungen der Vergleichsterne bekannt geworden. Die so erhaltenen Verbesserungen der Oerter veranlassten mich zu einer Wiederholung der Rechnung. Bei derselben wurden die folgenden Vergleichstern-Positionen benutzt:

\begin{tabular}{|c|c|c|c|}
\hline$*$ & $\alpha .878 .0$ & $\delta 1878.0$ & Autorität \\
\hline$a$ & $9^{\mathrm{h}} 2 \mathrm{I}^{\mathrm{m}} 53^{\mathrm{s}} \mathrm{I} 3$ & $+12^{\circ} 43^{\prime} 4^{\prime \prime \prime} \cdot 1$ & 2 Pola Mer. \\
\hline$b$ & $\begin{array}{lll}9 & 26 & 2.35\end{array}$ & +12 1622.5 & $1 / 2$ (Cap + Pola $)$ \\
\hline$c$ & $922 \quad 37.25$ & t 124942.5 & 2 Pola Mer. \\
\hline$d$ & 9 I $934.8 \mathrm{r}$ & +132143.0 & $1 / 3$ (2 Pola +Kremsm.) \\
\hline$\epsilon$ & 91146.89 & +153120.7 & 2 Pola Mer. \\
\hline$f$ & 91124.67 & +16352.9 & $1 / 2($ Pola + Berlin $)$ \\
\hline$g$ & $9 \quad 4 \quad 25.77$ & $\begin{array}{r}+19337.4 \\
\end{array}$ & $\begin{array}{l}1 / 5(2 \text { Pola }+2 \text { Berlin }+ \\
\text { Kremsm.) }\end{array}$ \\
\hline$h$ & 85945.60 & $\begin{array}{lll}121 & 0 \quad 10.9\end{array}$ & $\begin{array}{l}1 / 4 \text { (3 Result. aus Lal., B.Z., } \\
\text { Y.u. A. N. } 2008+\text { Knopf) }\end{array}$ \\
\hline$i$ & $9 \circ 57.09$ & $+2123 \times 2.3$ & $1 / 3$ (2 Pola+Kremsm.) \\
\hline
\end{tabular}

Die AR. des Sterns $g$ ist unrichtig, weil die Beobachtungen aus Pola und Berlin mit der in den A. N. 2337 für AR. unrichtig angegebenen Präcession auf 1878.0 reducirt sind; dieselbe sollte heissen: $\alpha=9^{\mathrm{h}} 4^{\mathrm{m}} 25^{\mathrm{s}} 34$.

Die Rechnung gründet sich auf die folgenden Normalörter:

\begin{tabular}{|c|c|c|c|c|c|c|c|}
\hline & 1878 & \multicolumn{2}{|c|}{$\alpha 1878.0$} & \multicolumn{3}{|c|}{$\delta \times 878.0$} & Gw. \\
\hline Feb & & $140^{\circ} 21$ & ' 2:6 & & 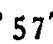 & & 3 \\
\hline & $2 \mathrm{x}$ & I37 55 & 23.0 & +15 & 48 & 50.1 & 2 \\
\hline & $\begin{array}{r}9.5477 I \\
24.50000\end{array}$ & $\begin{array}{l}135 \\
\text { I34 }\end{array}$ & $\begin{array}{l}19.7 \\
33.7\end{array}$ & $\begin{array}{l}+19 \\
+21\end{array}$ & $\begin{array}{r}4 \\
10\end{array}$ & $\begin{array}{r}23.4 \\
4.6\end{array}$ & \\
\hline
\end{tabular}

Als Resultat erhielt ich die im Berliner Jahrbuch für 1890 publicirten Elemente:

Epoche 1878 März 2.5 M. Z. Berlin.

$$
\begin{aligned}
& M=58^{\circ} 39^{\prime} 57^{\prime \prime} 5 \\
& \omega=262 \quad 17 \quad 9.2 \\
& \Omega=14245 \text { 57.I Mittl. Aequ. } 1878.0 \\
& i=263036.2 \\
& \varphi=203320.0 \\
& \mu=75^{6: " 9917} \\
& \log a=0.447277
\end{aligned}
$$

Die Darstellung der Normalörter war wie folgt:

$$
\begin{array}{cc}
\Delta \lambda \cos \beta & \Delta \beta \\
0.0 & -0.9 \\
+0.2 & +2.1 \\
-0.8 & -\mathrm{I} .7 \\
+0.3 & +0.2
\end{array}
$$

Die übrigbleibenden Fehler erscheinen hier bereits mit der Quadratwurzel des Gewichts multiplicirt. Die Summe der Fehlerquadrate war 9.3 Bogensecunden.

Den Herren Palisa und Knorre wurden nun Ephemeriden zugestellt, welche sowohl diesen Elementen zugehörten, als auch denjenigen, welche den folgenden Variationen in $\mu$ entsprachen: $\delta \mu=-3.552, \delta \mu=-1.932$, $\delta \mu=+1.828, \delta \mu=+3.608$. Dr. Palisa hat an einem Abend im December $x 886$ die Gegend zwischen der ersten und zweiten Hypothese mappirt. Da gleich nachher verschiedene neu entdeckte Planeten seine Aufmerksamkeit in Anspruch nahmen, wurde er an der weiteren Nachforschung verhindert. 
Dr. Luther hat bei seiner Berechnung mehr Bestimmungen der Vergleichsterne zur Verfügung gehabt. Auch ist die Summe der Fehlerquadrate bei ihm wesentlich verkleinert. Jedoch möchte ich nicht auf diese Umstände allzu viel Gewicht, was die erhöhte Sicherheit des Resultats betriff, legen. Einestheils weil die Differenzen Planet-Stern wenigstens theilweise mit bedeutender Unsicherheit behaftet zu sein scheinen, wie z. B. aus den zwei auf gleiche Zeit reducirten Bestimmungen Febr. 8 hervorgeht, welche ergeben:

$$
\begin{array}{cc}
\alpha \text { app. } & \delta \text { app. } \\
9^{\mathrm{h}} 22^{\mathrm{m}} 5^{3} .14 & +12^{\circ} 3^{\prime} 5^{88^{\prime \prime} 9} \\
49.22 & 3312.6
\end{array}
$$

Andererseits ist auch eine ziemlich grosse Unsicherheit daraus zu erwarten, dass die Correctionen der 6 Elemente aus nur 8 Bedingungsgleichungen bestimmt werden müssen, von denen 2 nur auf je einer einzelnen Beobachtung beruhen.
Ich bin deshalb der Meinung, dass, wenn ein Erfolg erwartet werden soll, der Aufsuchungszone eine ziemlich bedeutende Ausdehnung gegeben werden muss. Die von Herrn Dr. W. Luther angegebenen Elemente fallen sehr nahe mit denjenigen zusammen, welche der einen der bei Dr. Palisa's erstem Suchen benutzten Grenzephemeriden entsprechen. Bei einer eventuellen Nachforschung würde es sich deshalb empfehlen, nicht sich so sehr in der Gegend zu halten zwischen den Oertern aus Dr. Luther's Ephemeride und denjenigen aus meiner Jahresephemeride im Berliner Astr. Jahrbuch, sondern hauptsächlich die Gegend zu berücksichtigen, welche noch ausserhalb dieser und zwar nach der Seite der Ephemeride von Dr. Luther hin liegt. Für das dafür nothwendige Extrapoliren bietet das dort angegebene $\Delta \delta$ bei angemessen gewähltem $\Delta \alpha$ eine Hülfe.

Helsingfors 1888 März 27.

\section{Wiederaufitindung des Planeten (183) Istria.*)}

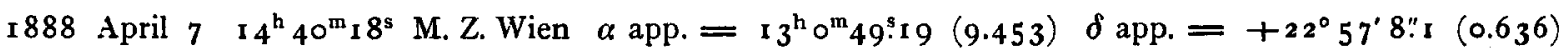

Correction der Ephemeride (A. N. $283 \mathrm{I}$ ): $+4^{\mathrm{m}} 39^{5}$ in AR. und -8.6 in Decl.

Wien 1888 April 8.

F. Palisa.

*) Eine von Dr. W. Luther auf Grund dieser Beobachtung vorgenommene Verbesserung seiner Ephemeride in A. N. 28.31 wird imnächsten Berl. Circ. (Nr. 317 ) erscheinen. Ktz.

\section{Entdeckung eines neuen Veränderlichen im Sternbilde Canes venatici.}

(Wolsingham Observatory Circular No. r9.)

The star DM. $+40.2694, \alpha=13^{\mathrm{h}} 4^{2^{\mathrm{m}}} 43^{\mathrm{s}} .2, \delta=+40^{\circ} 15: 9$ (1855), Mag. 9.2, was observed here on the nights April 6,8 as $7 \cdot 3$ and $7 \cdot 7$. It seems, therefore, variable. The spectroscope shows a fine III type spectrum.

Wolsingham Observatory, 1888 April 9.

T. E. Espin.

Aufforderung betr. Beobachtungen des Cometen 1887 III (Barnard Febr. 16).

Herr Mag. P. A. Heinricius (Sternwarte Helsingfors), der die Bearbeitung des Materials betreffend diesen Cometen zu übernehmen beabsichtigt, bittet etwa noch nicht publicirte Beobachtungen baldigst nittheilen zu wollen.

Anders Donner.

Beobachtungen des Cometen 1887 IV

\begin{tabular}{|c|c|c|c|c|c|c|c|c|c|c|c|c|c|}
\hline \multicolumn{2}{|c|}{1887} & M. Z.Genf & $\Delta a$ & \multicolumn{2}{|r|}{$\Delta d$} & $\mathrm{Vgl}$ & \multicolumn{2}{|c|}{$\alpha$ app. } & $\log p \cdot 4$ & $\delta$ app. & $\log p .4$ & Red.ad l.app. & $*$ \\
\hline Juli & $\begin{array}{l}6 \\
6\end{array}$ & $\begin{array}{l}10^{\mathrm{h}} 15^{\mathrm{m}} 54^{\mathrm{s}} \\
102624\end{array}$ & $-\mathrm{I}^{\mathrm{m}} 12.6 \mathrm{I}$ & & $\begin{array}{ll}6^{\prime} & 5^{\prime \prime} 9\end{array}$ & $\begin{array}{l}9 \\
3\end{array}$ & & $4^{m} 37^{s} \cdot 5 \mathrm{r}$ & 8.171 & $+4^{\circ}{ }^{-} 6^{\prime} 4^{\prime \prime} \mathrm{I}$ & 0.766 & $\begin{array}{c}+2^{5} x I \\
-\end{array}$ & $\begin{array}{l}\mathbf{I} \\
\mathbf{I}\end{array}$ \\
\hline & 7 & $\begin{array}{lll}9 & 43 & 15\end{array}$ & to 11.69 & - & I 11.6 & 12.6 & & 一 & $8.574 \mathrm{n}$ & - & 0.763 & $+2.10+9.0$ & 2 \\
\hline & 8 & $9 \quad 58 \quad 47$ & +135.05 & - & $\begin{array}{ll}6 & 27.9\end{array}$ & I 2.4 & & 一 & 7.854 & - & 0.761 & $+2.10+9.2$ & ) \\
\hline & I I & $957 \quad 1$ & to 4.10 & - & $\begin{array}{ll}524.7 \\
\end{array}$ & 20.10 & 17 & 1524.26 & $6.840_{n}$ & +62743.7 & 0.753 & $+2.11+9.9$ & 4 \\
\hline & 12 & $\begin{array}{lll}9 & 40 & 43\end{array}$ & to 55.84 & + & $4 \quad 17.0$ & 17.10 & 17 & 1730.49 & $8.430_{n}$ & +64254.8 & 0.750 & $+2.11+10.1$ & 5 \\
\hline & 14 & 10 2046 & to 50.09 & + & $\begin{array}{ll}4 & 46.5\end{array}$ & 12.6 & & - & 8.712 & - & 0.746 & $+2.12+10.6$ & 6 \\
\hline & 16 & 10737 & -o 19.18 & & - & 6 & & - & 8.548 & - & - & +2.12 & 7 \\
\hline & 16 & 10 1049 & - & - & 350.0 & 5 & & - & - & - & 0.742 & +11.0 & 7 \\
\hline & 19 & $947 \quad 17$ & to 4.49 & + & I 12.2 & 16.10 & & - & 7.896 & - & $0.73^{8}$ & $+2.13+11.6$ & 8 \\
\hline & 23 & $9353^{6}$ & 5.83 & - & 437.7 & I8.6 & & - & 7.6 & - & 0.733 & $+2.13+12.4$ & 9 \\
\hline Aug. & 8 & $\begin{array}{lll}9 & 41 & 34\end{array}$ & -030.81 & + & 457.0 & I 4.6 & & - & 8.834 & - & 0.731 & $+2.15+15.1$ & IO \\
\hline
\end{tabular}

ausgeführt am Zehnzöller der Sternwarte zu Genf von $A$. Kammermann.

(Fortsetzung.) 